\title{
Determining the thermomechanical image shift for the MIGHTI instrument on the NASA-ICON satellite (Erratum)
}

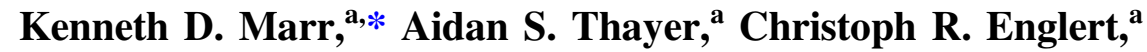 \\ and John M. Harlander ${ }^{\text {b,c }}$ \\ ${ }^{a}$ Space Science Division, U.S. Naval Research Laboratory, Washington, DC, United States \\ ${ }^{b}$ St. Cloud State University, Department of Physics, St. Cloud, Minnesota, United States \\ ${ }^{\mathrm{c}}$ Space Systems Research Corporation, Alexandria, Virginia, United States
}

[DOI: 10.1117/1.OE.60.3.039802]

This article [Opt. Eng. 59(1), 013102 (2020) https://doi.org/10.1117/1.OE.59.1.013102] was originally published on 11 January 2020 with a typographical error that affected some of the reported results. In several instances " $10,000^{\text {th }}$ " was given incorrectly. The value should have appeared as " $1000^{\text {th }}$." The value " 10,000 " has been corrected to " 1000 " in the Introduction, Methodology, and Results and Discussion sections, including Figs. 6-8. The Results and Discussion section also has had some of the wind speed numbers revised accordingly.

The paper was corrected online on 11 March 2021.

*Address all correspondence to Kenneth D. Marr, E-mail: kenneth.marr@nrl.navy.mil 